\title{
Modelling and measurement of UHF radio propagating through floors in a multifloored building
}

\author{
J.H.Tarng \\ D.W.Perng
}

Indexing terms: UHF radio propagation, Floors, Theoretical models, Multifloored buildings

\begin{abstract}
A general three-dimension theoretical model is developed to predict the path loss of UHF radio propagation through floors in a multifloored building. The computed propagation loss is compared with the measured value of $900 \mathrm{MHz}, 1.4 \mathrm{GHz}, 1.9 \mathrm{GHz}$, and $2.44 \mathrm{GHz}$ and gives a reasonable agreement. It is found that the floor, lateral beams and the walls around the receiver are the major construction features to affect the estimation of the penetration loss through floors.
\end{abstract}

\section{Introduction}

Radio propagation in the UHF band has been proposed as a basis for radio local area networks(R-LAN) and personal communications services (PCS)[1-3]. These systems, such as DECT, CT2, CT3 and the Universal Portable Digital Communication(UPDC) in the United States, can find important applications inside large office buildings. Their implementations require an understanding of the signal inside such structures. Hence, there are a number of recent studies on indoor radio propagation modelling [4-13] to investigate how signal characteristics are strongly influenced by specific features such as the layout in a floor, the construction materials and the floor types. They have developed both empirically based statistical models and theoretical models.

In this paper, a three-dimensional (3-D) theoretical propagation model combined with the ray-fixed coordinates $[13,14]$ is developed to investigate the mechanisms governing the UHF radio wave propagating through neighbouring floors in multifloored buildings. There have been several studies [4-9] that dealt with related subjects before. In [4-8], the authors employed empirical attenuation models to determine the average floor attenuation. It seems that only W. Honcharenko, H. Bertoni and J. Dailing have developed a theoretical model to investigate mechanisms governing propagation between different floors in mutifloored buildings [9]. The authors discovered that two paths over which

\section{(C) IEE, 1997}

IEE Proceedings online no. 19971299

Paper first received 8th November 1996 and in revised form 24th March 1997

The authors are with the Department of Communication Engineering, National Chiao Tung University, Hsin-Chu, Taiwan, Republic of China propagation can take place are (1) paths that involve transmission through the floors, and (2) paths having segments outside the buildings and involving diffraction at window frames. It seems that they only considered the direct ray for the first kind of paths. The major concern of the authors is to find the penetration loss through floors, hence, only one received signal is measured in each floor and compared with the computed signal. It is hard to understand how the floor construction affects the penetration loss through a floor.

In our research, a more general three-dimensional theoretical model employing the ray-tracing technique is developed to evaluate the radio loss. The direct transmission ray as well as the rays that are multiply reflected and transmitted at walls and floors are considered. It is found that in addition to the direct transmission ray, the rays reflected both by the lateral beams and walls also have large effect on the receiving fields. The lateral beam is designed for building floor to carry heavy loads and frequently used in high-rise concrete buildings which are built for the purpose of public activity. With our model, the floor penetration loss can be evaluated reasonably accurate to compare with the measured data of $900 \mathrm{MHz}, 1.4 \mathrm{GHz}, 1.9 \mathrm{GHz}$ and $2.44 \mathrm{GHz}$ radio waves.

\section{Propagation models}

\subsection{Ray tracing technique}

The ray tracing technique is used to trace the significant direct (if existing), reflected, transmitted or refracted rays from a transmitter location to a receiver location in a three-dimensional space. The transmitting unit sphere is decomposed into many ray tubes. Each ray tube, chosen to have nearly equal shape and area, is represented by a source ray during the tracing process. The ray tracing is accomplished by an exhaustive search of a ray tree accounting for the decomposition of the ray at each building-object intersection $[10,11]$. The reflected and transmitted rays then treated separately in a similar fashion as a source ray. This recursion continues until the ray intensity falls below a specified threshold or no further intersections occur. The reception sphere $[10,11]$ is also used to determine whether the traced ray approaching to a receiving point is received. Here, the reception sphere radius is equal to $\gamma d / 2$, where $d$ is the unfolded total path length and $\gamma$ is the angular spacing between neighbouring rays at the source. 


\subsection{Ray-fixed coordinate systems}

To trace the ray in a three-dimensional space in buildings and to include the polarisations of the field, the transmission or reflection coefficient of the propagating field has to be represented by a $3 \times 3$ matrix in a global coordinate system in the case of oblique incidence. The components of the matrix sometimes are difficult and time consuming to determine. With a ray-fixed coordinate system introduced, it is reduced to a $2 \times 2$ matrix which is much easier to determine.

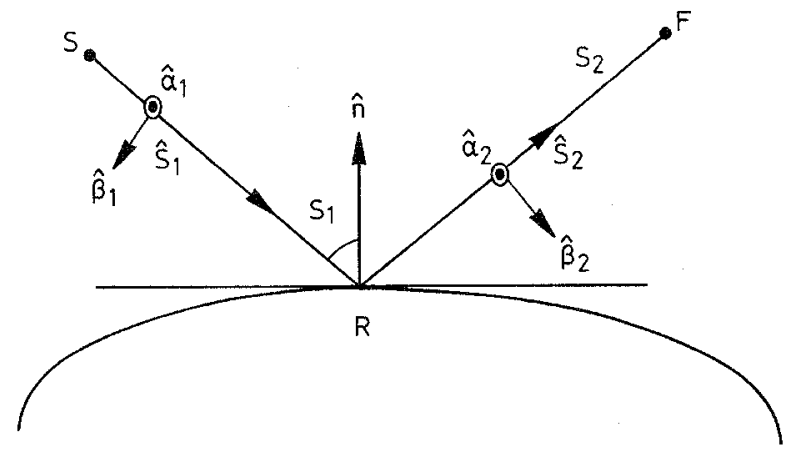

Fig. 1 Ray-fixed coordinate system associated with the reflection at the boundary

In Fig. 1, the incident and reflected ray-fixed coordinates, represented by $\left(\hat{s}_{1}, \hat{\alpha}_{1}, \hat{\beta}_{1}\right)$ and $\left(\hat{s}_{2}, \hat{\alpha}_{2}, \hat{\beta}_{2}\right)$, respectively, are the local coordinate systems. $\hat{s}_{1}$ and $\hat{s}_{2}$ are the unit vectors representing the directions of incident and reflected rays, respectively. The incident or reflected electric field at $R$ can always be resolved into components perpendicular $\left(\hat{\alpha}_{i}\right)$ and parallel $\left(\hat{\beta}_{i}\right)$ to the plane of incidence. Detail definitions of the both coordinate systems can be found in References [13, 14]. In the ray-fixed coordinate systems, the matrices of the reflection and transmission coefficients become, respectively,

$$
\overrightarrow{\vec{R}}_{c}=\left[\begin{array}{cc}
R_{s} & 0 \\
0 & R_{p}
\end{array}\right] \text { and } \overrightarrow{\vec{T}}=\left[\begin{array}{cc}
T_{s} & 0 \\
0 & T_{p}
\end{array}\right]
$$

Here, $R_{S}\left(T_{S}\right)$ and $R_{P}\left(T_{P}\right)$ are the reflection (transmission) coefficients of perpendicular and parallel polarisations, respectively, and they are determined by $\mathrm{ABCD}$ matrix [15] since the structure of walls or floors is layered.

\subsection{Propagation model}

The model includes direct, reflected and transmitted fields represented by the rays. Each propagation mechanism is treated separately. The complex vector field of the $i^{\text {th }}$ ray at the receiver is given by

$$
\vec{E}_{i}=\vec{E}_{0} \cdot G_{t i} \cdot G_{r i} \cdot L_{i} \cdot \prod_{j} \overrightarrow{\vec{R}}_{c}\left(\theta_{j i}\right) \cdot \prod_{m} \overrightarrow{\vec{T}}\left(\theta_{m i}\right)
$$

where $\vec{E}_{0}$ is the field 1 meter away from the transmitting antenna, $G_{t i}$ and $G_{r i}$ are the field-amplitude radiation patterns of the transmitting and the receiving antennas; and $L_{i}$ is the free-space path loss of the $i^{\text {th }}$ component. $\theta_{j i}$ and $\theta_{m i}$ are the $j$ th reflecting and $m$ th transmitting angles, respectively.

\section{Experimental sites, measurement set-up and results}

\subsection{Measurement setup and procedure}

Narrow-band $(\mathrm{CW})$ signal strength measurements were made at $900 \mathrm{MHz}, 1.4 \mathrm{GHz}, 1.9 \mathrm{GHz}$, and $2.44 \mathrm{GHz}$.
A $13 \mathrm{dBm}$ CW signal was transmitted by a half-wavelength dipole antenna at a height $1.2 \mathrm{~m}$ above the ground. The transmitting system, including a signal generator, a section of cable and the transmitting antenna, has been calibrated in an anechoic chamber to measure the one-metre transmitting field strength in free-space. The receiving antenna is also a half-wavelength dipole antenna (Anritsu MP651B and MP663A) with the same height. Both the transmitting and receiving antennas are vertically polarised during the measurement. The receiver (Advantest R3261A) can instantaneously measure the signal strength between $-30 \mathrm{dBm}$ to $-110 \mathrm{dBm}$ over a $10 \mathrm{KHz}, 100 \mathrm{KHz}$, $100 \mathrm{KHz}$ and $100 \mathrm{KHz}$ interval for $900 \mathrm{MHz}, 1.4 \mathrm{GHz}$, $1.9 \mathrm{GHz}$ and $2.44 \mathrm{GHz}$, respectively. The received data is acquired automatically by a personal computer with a GPIB card. At each measured position, the measured field strength is obtained by a spatial sector averaged over 16 grid subpoints with a quarter wavelength spacing between neighbouring points. To assure that the propagation channel is time stationary during the measurement, the measured data has been averaged on screen over 10 instantaneous sampled values.

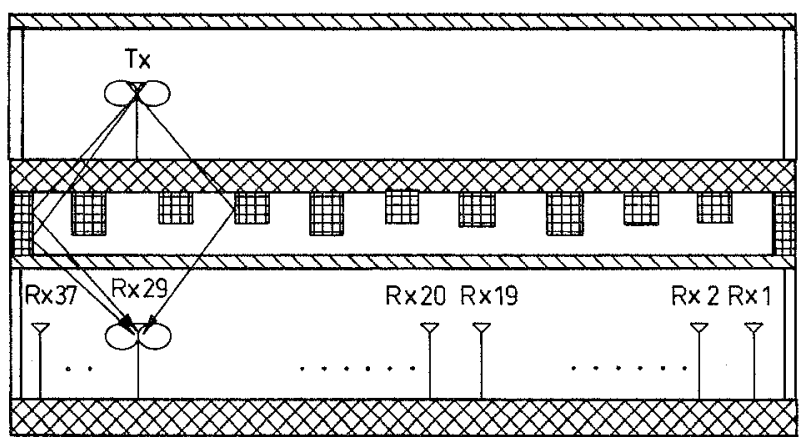

$$
\text { Yloor } \$ \text { ceiling lateral beam } \square \text { window }
$$

Fig.2 Cross-sectional view of experimental site NO.I which includes eighth and ninth floors of the building

$\mathrm{Tx}$ and $\mathrm{Rx}$ represent the positions of the transmitter and receiver, respectively. There are 37 measured positions chosen for this site

\subsection{Experimental sites}

The measurement site is at the eighth and ninth floors of Engineering Building Four at the National ChiaoTung University in Hsin-Chu. Fig. 2 shows a cross-sectional view of these floors at the south wing of the building. It is noted that there are several lateral beams between the floor and the ceiling with an unequal spacing between the neighbouring beams. These beams with finite width are extended very long and may have significant effect on the radio loss through the floor, which will be discussed later. In this research, two experimental sites are chosen where site NO.1 is the hallway at the south wing of eighth and ninth floors and site NO.2 includes room 913 and the hallway at the west wing of the building. In site NO.1 the transmitting antenna is fixed at the hallway of the ninth floor as shown in Fig. 2 and the receiving antenna is moving along the hallway with a total number of 37 positions being measured. These two floors have similar structure and construction materials. The width, height and length of both hallways are $2.6 \mathrm{~m}, 2.4 \mathrm{~m}$ and $34 \mathrm{~m}$, respectively. The side-walls of the hallway are mainly made of plaster board. The ground of the corridor is made of reinforced concrete. The major feature of the ceiling is defined by the lighting fixtures made of metal. $900 \mathrm{MHz}$ and $2.44 \mathrm{GHz}$ radio propagation loss 
through the floor is measured in site NO.1. Fig. 3 shows the top view of measurement site NO.2. The transmitting antenna is fixed and located at the lobby of the eighth floor. The receiving antenna in this experiment is not only moving along the hallway but also in Room 913 which are both at the ninth floor. The measured frequencies include $900 \mathrm{MHz}, 1.4 \mathrm{GHz}, 1.9 \mathrm{GHz}$ and $2.44 \mathrm{GHz}$ at site NO.2. During the measurement at both sites, both the transmitting and receiving antennas are vertically polarised.

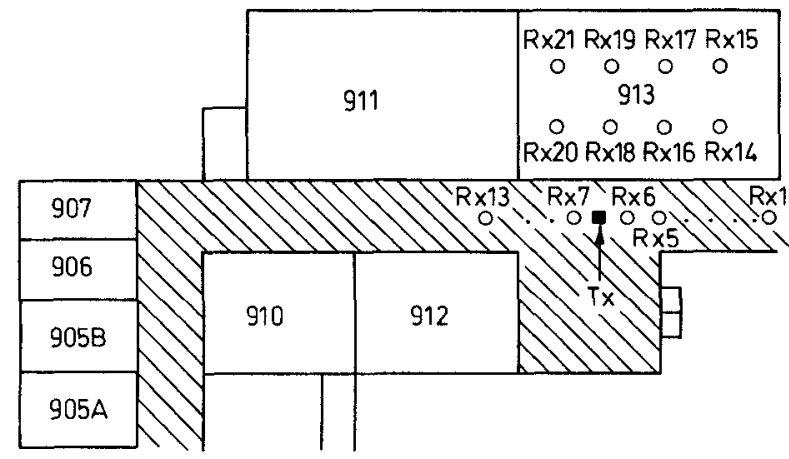

Fig. 3 The top view of measurement site NO.2

The transmitting antenna, marked with $\mathbf{\square}$, is located at eighth floor while the receiving antenna is moving at ninth floor. Total number of 21 positions are measured. The shaded area represents the region of corridor or lobby of the floor

\section{Comparisons}

In the numerical simulation made with the threedimensional model, a total of 16200 source rays are generated and traced. The dielectric constants of the ceiling, plaster-board and the window in the hallway are chosen to be equal to $5,5-\mathrm{j} 0.062$ and 6 , respectively [16]. For the concrete walls, the dielectric constants are equal to $7-\mathrm{j} 1.2,7-\mathrm{j} 0.964,7-\mathrm{j} 0.81,7-\mathrm{j} 0.74$ for $900 \mathrm{MHz}$, $1.4 \mathrm{GHz}, 1.9 \mathrm{GHz}$ and $2.44 \mathrm{GHz}$, respectively[17]. To compare the computed path loss with the measured one at site NO.1, in addition to the three-dimensional model, the DP (direct-path) model is also employed. The DP model considers the direct-transmission (refracted) ray only.

In Fig. 4, the measured path loss relative to onemetre field strength in free-space of $900 \mathrm{MHz}$ radio wave at site NO.1 is compared with that computed by the DP and three-dimensional models. There are total 37 receiving positions as shown in Fig. 2. It is found that the three-dimensional model gives a much better prediction accuracy than that of the DP model with smaller values of $m_{e}$ and $\sigma_{e}$ Here $m_{e}$ represents the mean of the error (the difference between the simulated and measured path losses) and $\sigma_{e}$ is the standard deviation of the error. It is because the field transmitted through the floor and reflected by the lateral beams or by the sidewalls in the hallway has a significant contribution to the receiving field, which is neglected in the DP model. This can be explained by the geometry of the received rays that are transmitted through the floor and reflected by the lateral beams as shown in Fig. 2. Therefore, for the receiving positions near to the transmitting antenna, for example $\mathrm{Rx} 27, \mathrm{Rx} 28, \mathrm{Rx} 29$, $\mathrm{Rx} 30$ and $\mathrm{Rx} 31$, the three-dimensional model gives much better prediction accuracy than that of the DP model as shown in Fig. 4 since in this region the fields reflected by the lateral beams or sidewalls are the major components of the receiving field. It is noted that the path loss predicted by the DP model of the receiving position number 29 , i.e., $\operatorname{Rx} 29$, approaches to $\rightarrow \infty$ in theory since the receiving antenna is right under the transmitting antenna and the null-to-null situation happens. Therefore values of the computed path loss of Rx 29 are not shown in Fig. 4. In Fig. 5 the path loss computed by the modified 3-D model is compared with the measured one. The modified model is the 3-D model neglecting the rays which are propagating through the floor and reflected by the sidewalls. Although this model only considers the direct-transmitted ray and the rays reflected by the lateral beams, it still gives a reasonable prediction accuracy with $m_{e}=-5.1 \mathrm{~dB}, \sigma_{e}=$ $4.8 \mathrm{~dB}$. By comparing the values of $m_{e}$ and $\sigma_{e}$ of the modified model with those of the $3-\mathrm{D}$ models, it is found that the floor and lateral beams are the major construction features to affect the estimation of the floor attenuation and the sidewall reflection can raise the predicted field strength.

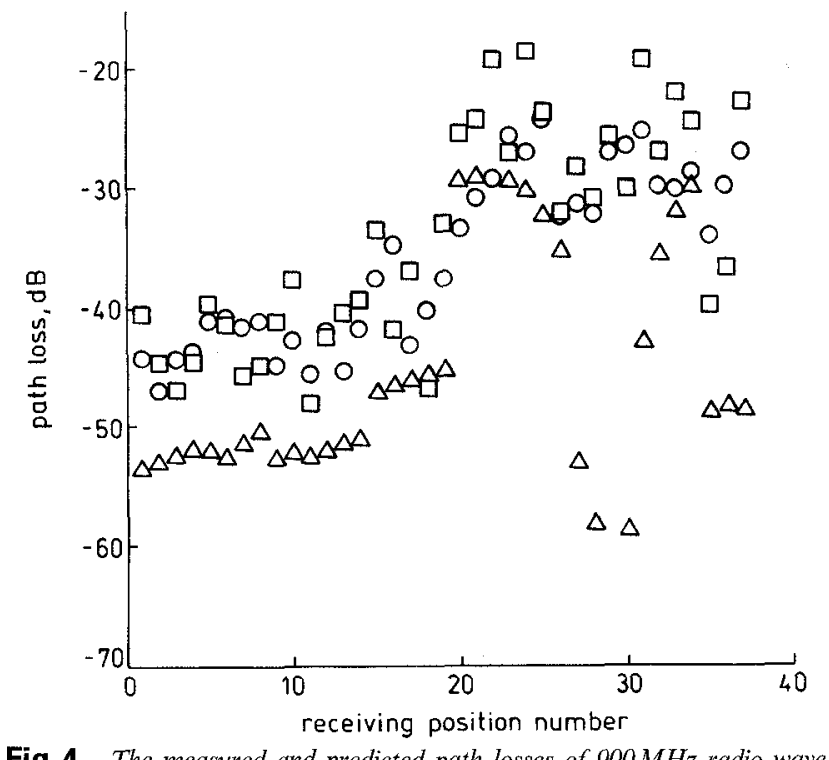

Fig.4 The measured and predicted path losses of $900 \mathrm{MHz}$ radio wave at site NO.1 as a function of number of the receiving position measured

$\square 3 \mathrm{D}$ model

$m_{e}=1.58 \mathrm{~dB} \sigma_{e}=4.49 \mathrm{~dB}$

$m_{e}=-9.32 \mathrm{~dB} \sigma_{e}=7.64 \mathrm{~dB}$

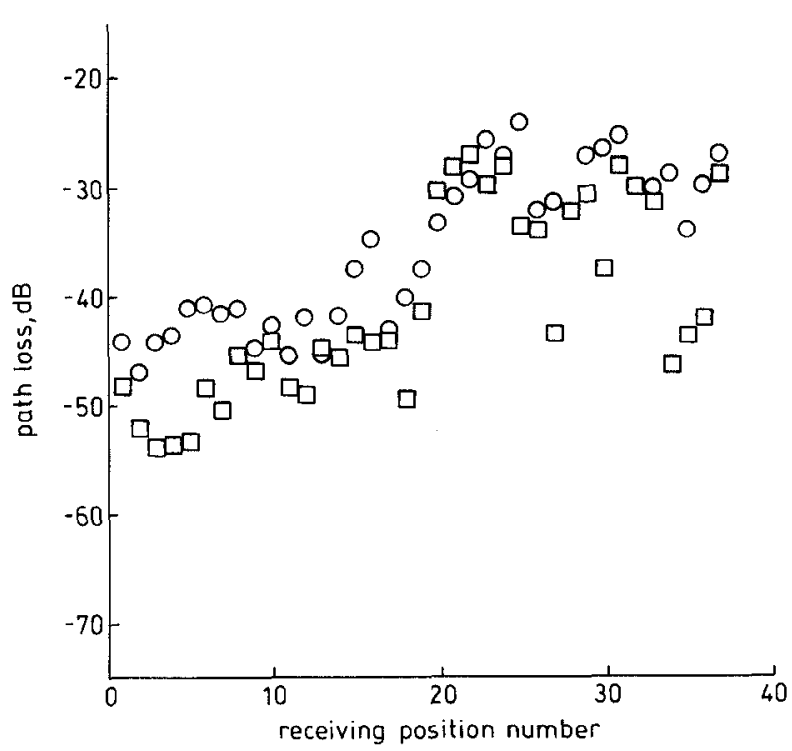

Fig.5 The measured and predicted path losses of $900 \mathrm{MHz}$ radio wave at site NO.I as a function of number of the receiving position

The predicted path loss is computed by using the 3-D model without including the rays that propagate through the floor and are reflected by the sidewalls 0 measured $\square$ modified model

$m_{e}=-5.12 \mathrm{~dB} \sigma_{e}=4.85 \mathrm{~dB}$ 
In Fig. 6, the measured path loss of $2.44 \mathrm{GHz}$ radio wave at site NO.1 is compared with that computed by the DP and the 3-D models. The 3-D model still gives a much better prediction accuracy with $m_{e}=1.6 \mathrm{~dB}$ and $\sigma_{e}=4.7 \mathrm{~dB}$ while $m_{e}=-9.2 \mathrm{~dB}$ and $\sigma_{e}=10.5 \mathrm{~dB}$ for the DP model.

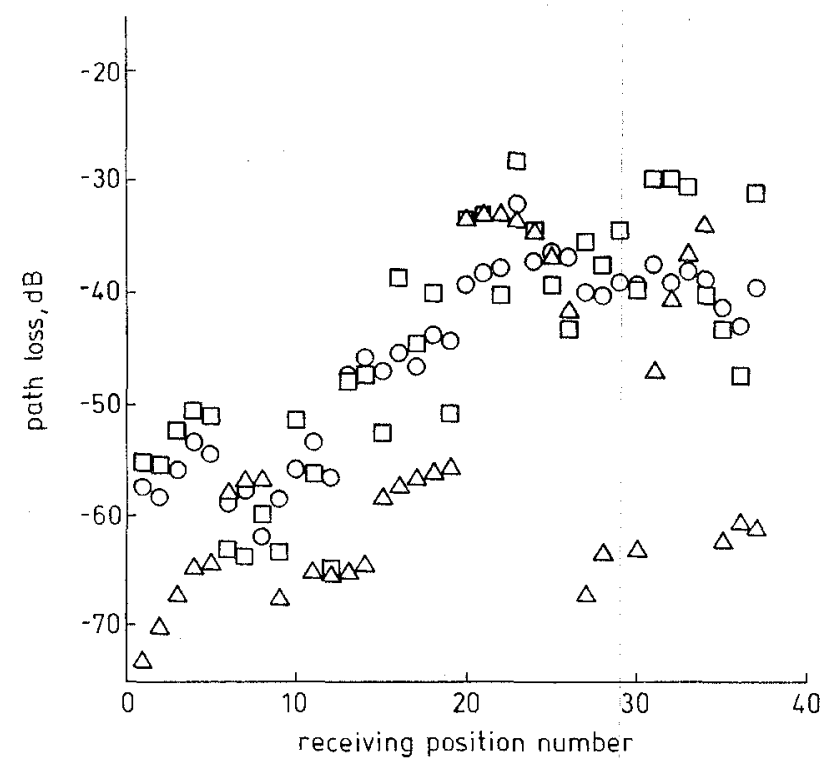

Fig.6 The measured and predicted path losses of $2.44 \mathrm{GHz}$ radio wave at site NO.I as a function of number of the receiving position

$O$ measured

$\square$ 3D model

$m_{e}=0.98 \mathrm{~dB} o_{e}=4.67 \mathrm{~dB}$

$m_{e}=-8.81 \mathrm{~dB} \sigma_{e}=9.39 \mathrm{~dB}$

To assure the prediction accuracy of the 3-D model, additional measurements of frequencies of $900 \mathrm{MHz}$ $1.4 \mathrm{GHz}, 1.9 \mathrm{GHz}$ and $2.44 \mathrm{GHz}$ are carried out at site NO.2 and the measured path loss is compared with the computed one. A total number of 21 positions as shown in Fig. 3 are measured for each frequency. By comparing the computed path loss with the measured, value of $\sigma_{e}$ for each frequency is illustrated in Table 1. It is shown that the 3-D model still gives a consistent prediction accuracy for various transmitting frequencies.

Table 1: Value of $\sigma_{e}$ for various at site NO.2

\begin{tabular}{ll}
\hline $\begin{array}{l}\text { Frequency } \\
(\mathrm{GHz})\end{array}$ & $\sigma_{e}(\mathrm{~dB})$ for 3-D model \\
\hline 0.9 & 4.85 \\
1.4 & 5.44 \\
1.9 & 5.27 \\
2.44 & 4.62 \\
\hline
\end{tabular}

To study the floor penetration loss extensively, a floor attenuation factor (FAF) path loss model proposed by [7] is used and the average FAF is estimated by the measurement data at site NO.1 and NO.2 for frequencies $900 \mathrm{MHz}$ and $2.44 \mathrm{GHz}$. The values of FAF are shown in Table 2. It is found that the values of FAF for two measurement sites are similar but larger than those in [7], which may be due to the differences of floor layout, construction features, local environments and number of sample points for fitting the regression line. It reveals the fact that the empirical model is used only for limited environments and is not possible to guarantee transportability of the prediction model between environments in which are classified as similar. On the other hand, the theoretical model, for example the one proposed in the paper, can provide accurate prediction as well as broad usage.

Table 2: Values of FAF for frequencies $900 \mathrm{MHz}$ and $2.44 \mathrm{GHz}$ at sites NO. 1 and NO.2

\begin{tabular}{lll}
\hline Site No. & $\begin{array}{l}\text { Frequency } \\
(\mathrm{GHz})\end{array}$ & FAF $(\mathrm{dB})$ \\
\hline 1 & 0.9 & -21 \\
1 & 2.44 & -26.91 \\
2 & 0.9 & -22 \\
2 & 2.44 & -30.91 \\
\hline
\end{tabular}

\section{Conclusion}

A general three-dimensional model is developed and gives a reasonable prediction accuracy for $900 \mathrm{MHz}$, $1.4 \mathrm{GHz}, 1.9 \mathrm{GHz}$ and $2.44 \mathrm{GHz}$ radio waves to propagate through floors in a multifloored office building. It is found that the receiving field is governed by the direct transmission path as well as the paths transmitted through the floor and reflected by the lateral beams or by the sidewalls. The floor and lateral beams are the major construction features governing the floor attenuation and the sidewall reflection may raise the predicted field strength.

\section{Acknowledgment}

This research is supported by the National Science Council of Taiwan under the project number: NSC 852221-E-009-35.

\section{References}

1 MARCUS, M.: 'Regulatory policy considerations for radio local area networks', IEEE Commun. Mag., July 1987, 25, (7), pp. 95 99

2 HASHEMI, H.: 'The indoor radio propagation channel', Proc IEEE (USA), July 1993, 81, (7), pp. 943-968

3 MOLKDAR, D.: 'Review on radio propagation into and within buildings', Proc. Inst. Elec. Eng. (UK) Pt. H, Feb. 1991, 138, pp. $61-73$

4 LAFORTUNE, J.F., and LECOURS, M.: 'Measurement and modeling of propagation losses in a building at $900 \mathrm{MHz}$, IEEE Trans. Veh. Technol. (USA), May 1990, 39, (2), pp. 101-108

5 MOTLEY, A.J., and KEENAN, J.M.P.: 'Radio coverage in buildings', British Telecom. Tech. J., Special Issue on Mobile Communications, 1990,8 , pp. 19-24

6 MOLEY, A.J., and KEENAN, J.M.P.: 'Personal communication radio coverage in buildings at $900 \mathrm{MHz}$ and $1700 \mathrm{MHz}$, Electron. Lett., June 1988, 24, pp. 763-764

7 SEIDEL, S.Y., and RAPPAPORT, T.S.: ' $914 \mathrm{MHz}$ path loss prediction models for indoor wireless communications in multifloored buildings', IEEE Trans. Antennas Propag., Feb. 1992, 40, pp. 207-217

8 HORIKOSHI, J., TANNAKA, K., and MORINAGA, T. '1, $\mathrm{GHz}$ band wave propagation measurements in concrete building for indoor radio communications', IEEE Trans. Veh. Technol. (USA), Nov. 1986, 35, (4), pp. 146-152

9 HONCHARENKO, W., BERTONI, H.L., and DAILING, J. "Mechanisms governing propagation between different floors in buildings', IEEE Trans. Antennas Propag., June 1993, 41, (6), pp $787-790$

10 SEIDEL, S.Y., and RAPPAPORT, T.S.: 'Site-specific propagation prediction for wireless in-building personal communication system design', IEEE Trans. Veh. Technol. (USA), Nov. 1994, 43, (4), pp. $879-892$

IEE Proc.-Microw. Antennas Propag., Vol. 144, No. 5, October 1997 
11 HONCHARENKO, W., BERTONI, H.L., DAILING, J., QIAN, J., and YEE, H.D.: 'Mechanisms governing UHF propagation on single floors in modern office buildings', IEEE Trans. Veh. Technol. (USA), Nov. 1992, 41, (4), pp. 496-504

12 WHITMAN, G.M., KIM, K.S., and NIVER, E.: 'A theoretical model for radio signal attenuation inside buildings', IEEE Trans. Veh. Technol. (USA), Aug. 1995, 44, (3), pp. 621-629

13 TARNG, J.H., CHANG, W.R., and HSU, B.J.: 'Three-dimensional modeling of $900 \mathrm{MHz}$ and $2.44 \mathrm{GHz}$ radio propagation in corridors', IEEE Trans. Veh. Technol. (USA), May 1997, 46, (2), pp. $519-527$
14 McNAMARA, D.A., PISTORIUS, C.W.I, and MALHERBE, J.A.G.: 'Introduction to the uniform geometrical theory of diffraction' (Artech House, Boston, 1990), p.69

15 ISHIMARU, A.: 'Electromagnetic wave propagation, radiation, and scattering' (Prentice-Hall, NJ, 1991), Chap. 3

16 COLLIN, R.E., and ZUCKER, F.J.: 'Antenna theory' (McGraw-Hill, New York, 1969)

17 YANG, C.F., KO, C.J., and CHEN, J.Y.: 'Measurements of the dielectric constants of the walls in buildings'. Proceedings of the first Radio science symposium, 1995, pp. 63-67 